\title{
Proteinopathies: molecular mechanisms and diagnostic perspectives
}

\author{
C. Delaby ${ }^{1,2} \cdot$ S. Lehmann ${ }^{3}$
}

Accepted: 20 January 2022 / Published online: 25 January 2022

(c) The Author(s), under exclusive licence to Springer-Verlag GmbH Austria, part of Springer Nature 2022

Proteinopathies are diseases associated with conformational changes in proteins. These changes lead to an alteration of their function, most often related to the formation of oligomers, which can then aggregate into higher order pathological structures. It is important to note that these aggregated forms of misfolded proteins have the ability to induce the conversion of normal proteins, a mechanism supporting the transmissibility of these diseases. Many neurodegenerative diseases, such as Alzheimer's disease (AD), Parkinson's disease (PD) or Creutzfeldt-Jakob disease (CJD), belong to this group of pathologies and are characterized by the accumulation in neurons or in the brain parenchyma of specific pathological proteins (prion, synuclein, tau, amyloid. etc.). Given the importance of these diseases for public health, both in terms of prevalence and risk, it is important to understand their pathophysiology, their transmission and to be able to detect them in patients in an efficient way, notably through biological markers.

Among proteinopathies, prion diseases represented mostly by CJD, are unique by their high propensity to be transmissible. CJD represents, therefore, a public health problem, with a high risk of secondary iatrogenic transmission. Screening for the disease is, therefore, essential and can be based on different techniques of varying sensitivity

C. Delaby

c-delaby@chu-montpellier.fr;

constance.delaby@umontpellier.fr;constance.delaby@inserm.fr

S. Lehmann

s-lehmann@chu-montpellier.fr

1 Inserm U1298, CHU de Montpellier - Hôpital St. Eloi, Institut des Neurosciences de Montpellier (INM), Maître de Conférences Universitaire-Université de Montpellier, Montpellier, France

2 Biochimie - Protéomique Clinique, Institut de Médecine Régénératrice et de Biothérapie (IRMB), 80, avenue A. Fliche, 34295 Montpellier Cedex 5, France

3 Laboratoire de Biochimie Protéomique Clinique-PPC, Hôpital St Eloi, Univ Montpellier, CHU Montpellier, INM INSERM, IRMB 80 av A Fiche, 34295 Montpellier, France and specificity. The work of Fayolle M. et al. compares the diagnostic performance of CSF biomarkers (tau, tau/p-tau (181) and alpha-synuclein proteins) with that of 14-3-3 protein (gold standard for the diagnosis of CJD) in a cohort of suspected CJD patients. The results illustrate the high efficiency of tau protein detection for routine screening of the disease in a clinical setting (reaching 78\% sensitivity and $80 \%$ specificity for the detection of CJD), which allows for rapid and accurate results and thus allows confirmatory tests such as RT-QuIC to be performed without delay.

Alteration of protein folding in proteinopathies can impaired clearance mechanisms. As presented in the review by Vilchez D. et al., clearance of damaged and aggregated proteins is capable of ameliorating neurodegenerative disease-related changes in cellular and organizational models. Indeed, mechanisms such as the ubiquitin-proteasome system (UPS), autophagy, and aggregated proteins themselves could be a potential target for treating these diseases, as explained in Vilchez D. et al. The challenge now is to understand how these clearance mechanisms can be potentially modified to prevent disease.

Some proteinopathy disorders (such as Huntington's disease or frontotemporal dementia/amyotrophic lateral sclerosis associated with an expansion in the C9ORF72 gene) arise from genetic abnormalities such as unstable single sequence repeats (microsatellites) scattered throughout the human genome. These so-called repeat expansion disorders differ in the nature and location of the expanded segments, as summarized by Fourier A. et al. The presence of an expanded segment in a specific gene thus triggers diverse molecular mechanisms (at the DNA, RNA and protein levels), leading to neurodegeneration and disease onset. Their article summarizes how the improved understanding of these different mechanisms has led to recent advances in innovative therapeutic approaches and the identification of associated biomarkers.

Synapse degeneration represents the crucial neuropathological event in synucleinopathies, such as PD, dementia with Lewy bodies (DLB), and multiple systems atrophy (MSA). In these pathologies, $\alpha$-synuclein exists in a 
thermodynamic equilibrium of soluble oligomers and insoluble fibrillar forms. Aggregation of $\alpha$-synuclein at synapses is thought to be a major event in the pathogenesis of the synucleinopathies, leading to functional impairment of the connectome. In the review of Belbin O. et al., the authors revisit published findings related to candidate biomarkers of synapse degeneration in biofluids derived from patients with PD, DLB, and MSA and discuss the results in the context of mechanisms associated with $\alpha$-synuclein-mediated synapse degeneration.

Intensive research is currently underway to improve the understanding of the pathomechanisms involved in proteinopathies. Proteins belonging to the neuronal pentraxin (NPTX) family are progressively emerging as candidate biomarkers of synaptic impairment in various neurodegenerative diseases, although their pathophysiological role in neurodegeneration remains to be fully elucidated. These extracellular scaffolding proteins are involved in homeostatic synaptic plasticity by recruiting postsynaptic receptors to synapses. Thus dynamic changes of NPTX in cerebrospinal fluid (CSF) may reflect synaptic damage, possibly linked to cognitive impairment. The potential involvement of NPTX in synaptic damage and neurodegeneration is discussed in the review by Nerea Gómez de San José et al. The evaluation of their potential as CSF biomarkers for neurodegenerative diseases is also reviewed.

With respect to the diagnosis of $\mathrm{AD}$, the characterization of biomarkers with high sensitivity and specificity is undoubtedly essential for patient management. Amyloid and tau biomarkers in CSF are one of the criteria of international guidelines, but the use of CSF to detect $\mathrm{AD}$ at an early stage in a large population remains difficult due to the invasive nature of lumbar puncture. Blood biomarkers are of particular interest in this context and Delaby C. et al. evaluate in their work the performance of blood biomarkers (amyloid peptides, P-tau181) in the detection of "pathological" and "non-pathological" CSF profile. The results show that these biomarkers can differentiate these profiles with a sensitivity of $85.0 \%$ and a specificity of $86.7 \%$ and could therefore be decisive in the realization or not of a lumbar puncture. Blood biomarkers may, therefore, represent the first step in the patient management strategy to determine whether or not further diagnostic tests by more invasive or expensive means are necessary.

With regard to the differential diagnosis of proteinopathies, there is growing evidence of the informativeness of phosphorylated tau isoforms in plasma (P-tau181, P-tau217, and P-tau231) in distinguishing patients with Alzheimer's disease (AD) from subjects without cognitive impairment, but also from patients with other dementias. These features, along with the minimally invasive nature, scalability, and ease of longitudinal sampling, make these proteins promising peripheral biomarkers for AD in both research and clinical settings, as resumed by Verde F. The review also summarizes the main findings regarding these same proteins in the field of genetic $\mathrm{AD}$ and other proteinopathies.

All together, these articles/reviews provide an overview of the literature currently available in the field of proteinopathies, both at the mechanistic level, but also at the diagnostic management level of these diseases.

Publisher's Note Springer Nature remains neutral with regard to jurisdictional claims in published maps and institutional affiliations. 